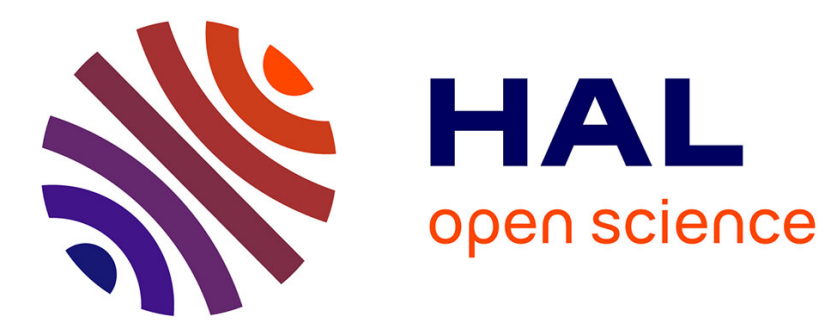

\title{
Numerical analysis of pitching-rotor aerodynamics
}

Thierry Jardin, Nicolas Doué, Sébastien Prothin, Jean-Marc Moschetta

\section{To cite this version:}

Thierry Jardin, Nicolas Doué, Sébastien Prothin, Jean-Marc Moschetta. Numerical analysis of pitching-rotor aerodynamics. Journal of Fluids and Structures, 2016, vol. 62, pp. 175-186. 10.1016/j.jluidstructs.2016.01.011 . hal-01446186

\section{HAL Id: hal-01446186 \\ https://hal.science/hal-01446186}

Submitted on 25 Jan 2017

HAL is a multi-disciplinary open access archive for the deposit and dissemination of scientific research documents, whether they are published or not. The documents may come from teaching and research institutions in France or abroad, or from public or private research centers.
L'archive ouverte pluridisciplinaire HAL, est destinée au dépôt et à la diffusion de documents scientifiques de niveau recherche, publiés ou non, émanant des établissements d'enseignement et de recherche français ou étrangers, des laboratoires publics ou privés. 


\section{Open Archive TOULOUSE Archive Ouverte (OATAO)}

OATAO is an open access repository that collects the work of Toulouse researchers and makes it freely available over the web where possible.

This is an author-deposited version published in: http://oatao.univ-toulouse.fr/ Eprints ID: 16628

To cite this version: Jardin, Thierry and Doué, Nicolas and Prothin, Sebastien and Moschetta, Jean-Marc Numerical analysis of pitching-rotor aerodynamics. (2016) Journal of Fluids and Structures, vol. 62. pp. 175-186. ISSN 0889-9746

Official URL: http://dx.doi.org/10.1016/j.jfluidstructs.2016.01.011

Any correspondence concerning this service should be sent to the repository administrator: staff-oatao@ listes-diff.inp-toulouse.fr 


\title{
Numerical analysis of pitching-rotor aerodynamics
}

\author{
T. Jardin *, N. Doué, S. Prothin, J.M. Moschetta \\ Institut Supérieur de l'Aéronautique et de l'Espace (ISAE-SUPAERO), Université de Toulouse, 31055 Toulouse Cedex 4, France
}

Keywords:

Flapping-rotor

Reversed von Karman

Dynamic stall

Leading-edge vortex

Micro-air vehicles

\begin{abstract}
A B S T R A C T
The influence of periodic blade pitching on rotor aerodynamics is numerically investigated at a Reynolds number typical of micro-air vehicles. Blade pitching motion is parameterized using three variables, giving rise to a large parameter space that is explored through 74 test cases. Results show that a relevant tuning of pitching variables can lead to an increase in rotational efficiency and thrust, which is found to be primarily related to the occurrence of reversed von Karman street, leading edge vortex (LEV) formation and dynamic stall phenomenon. In addition, for cases where reversed von Karman street occurs, the flow is found to be quasi-two-dimensional, suggesting that quasi-twodimensional approaches can provide relevant approximations of the global aerodynamics. Overall, the analysis demonstrates that blade pitching can be beneficial to the aerodynamic performance of micro-air vehicles and helps draw guidelines for further improvements of flapping-rotor concepts.
\end{abstract}

\section{Introduction}

Micro- (MAVs) and nano-air vehicles (NAVs) operate at Reynolds numbers roughly ranging from $10^{3}$ to $10^{5}$. At these Reynolds numbers, aerodynamic performance of conventional fixed and rotary wing concepts drastically decreases due to the increased importance of flow viscous forces with respect to inertial forces. Basically, increased viscous effects tend to increase viscous drag and to promote flow separation, which leads to a reduction in airfoil efficiency and maximum achievable lift respectively. Early examples of such reduced aerodynamic performance on NACA airfoils can be found in Jacobs and Sherman (1937). Reduction in airfoil efficiency and maximum achievable lift results in low endurance and limited payloads. Such issues significantly restrict the spectrum of applications, in a worldwide rapidly growing market. Therefore, the question is how can we tackle those issues?

The observation of nature can potentially bring an answer to this question. Indeed, conversely to conventional aircraft and helicopters which operate in the 'attached flow' regime, insect wings, for example, operate in the 'separated flow' regime. Flow separation is detrimental to conventional aircraft and helicopters but can be beneficial to insects, birds or fishes, where the resulting formation of vortices is used as high lift and locomotion mechanisms. Overall, two principal mechanisms emerge.

First mechanism is referred to as dynamic stall. When a high (fixed) angle of attack airfoil is impulsively started from rest in a quiescent fluid, the flow separates at the leading edge and rolls up into a leading edge vortex (LEV). The latter induces a low pressure region on the upper surface of the airfoil, which enhances lift. However, such a vortex is generally not stable in that its growth up to a critical size triggers its shedding from the airfoil, hence causing a drastic drop in the aerodynamic lift.

\footnotetext{
* Corresponding author.

E-mail address: thierry.jardin@isae.fr (T. Jardin).
} 
(a)

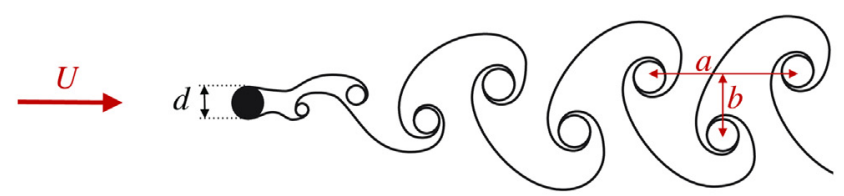

(b)

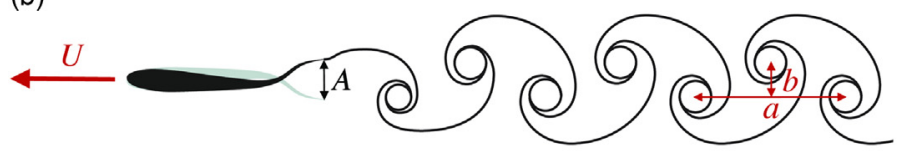

(c)

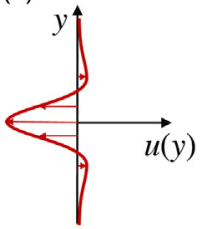

(d)

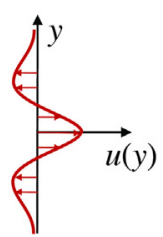

Fig. 1. Illustration of von Karman (a) and reversed von Karman (b) vortex street (reproduced from Eloy, 2012).

Then, a new LEV is formed and the scenario repeats itself, leading to an unsteady oscillating lift and a wake pattern known as a von Karman vortex street. The lowest angle of attack at which such an unsteady scenario occurs is referred to as the static stall angle. The mean value of the oscillating lift is lower than the steady lift obtained at lower angle of attack, in the absence of flow separation and vortex shedding. An interesting feature here is that the first LEV that develops immediately after the impulsive start remains attached to the airfoil longer than subsequent LEVs do (Wang, 2000). Thus, a longer plateau in high lift is observed at the impulsive start. A direct outcome is that airfoil angle of attack can be tuned to benefit from this plateau. In other words, if the angle of attack is increased above the static angle of attack to generate a 'first' LEV and then reduced below the static angle of attack before LEV shedding occurs, the airfoil can benefit from lift plateau while avoiding drastic drops in lift. If such a change in airfoil angle of attack is repeated, the lift still exhibits an unsteady behavior but its mean value is relatively high, i.e. higher than the maximum lift achievable on a static airfoil. This dynamic stall mechanism is intrinsically unsteady as it results from the unsteady motion of the airfoil. A fascinating example of dynamic stall mechanism can be found in nature, where insects and birds tune their flapping wings kinematics to benefit from the LEV and thus generate enough lift to keep them aloft (Dickinson et al., 1999). In a more general sense, dynamic stall phenomenon usually occurs on wings that undergo rapid changes in effective angle of attack (e.g. retreating helicopter rotor blades and highly maneuverable aircraft) and has therefore been extensively studied in the literature (e.g. McCroskey, 1982; Visbal, 1990; Eldredge et al., 2009).

Second mechanism is referred to as reversed von Karman street. As previously mentioned, a high (fixed) angle of attack airfoil translating in a quiescent fluid experiences low lift and its wake exhibits a von Karman vortex pattern due to periodic shedding of leading (LEVs) and trailing edge vortices (TEVs). von Karman vortex street is associated with wake velocity deficit which is indicative of drag. This drag generating mechanism can be clearly correlated with the position and circulation of LEVs and TEVs, as illustrated in Fig. 1. Therefore, a high angle of attack translating airfoil experiences both low lift and high drag, hence low aerodynamic efficiency. However, the position and orientation of the shed vortices can be altered via a time variation of the airfoil angle of attack, turning the wake pattern into a reversed von Karman street. Such a pattern is associated with a wake velocity surplus which is indicative of a propulsive force (Fig. 1). Reversed von Karman street is an ubiquitous feature in nature and explains the locomotion of fishes.

Overall, dynamic stall and reversed von Karman street appear as unconventional high lift and locomotion mechanisms respectively. These result from a time variation in airfoil angle of attack, which is generally achieved through pitching and plunging unsteady motions. During the past decades, MAVs relying on dynamic stall mechanism were developed using flapping wings concept (e.g. the Nano Hummingbird from AeroVironment, Keennon et al., 2012; RoboBee from Harvard University, Wood, 2008). The flapping wings concept is a prospectively relevant solution for low Reynolds number hovering flight where conventional concepts exhibit very poor aerodynamic performance. However, a major drawback of flapping wings concept is that the flapping motion is characterized by stroke reversal phases (pronation and supination phases) where the wing velocity approaches zero. Although additional mechanisms such as wake capture or Kramer effect (Dickinson et al., 1999) tend to mitigate the effect of stroke reversal phases, the latter are still detrimental to aerodynamic performance. On the other hand, MAVs relying on the reversed von Karman street mechanism were also developed using flapping wings concept to achieve propulsion for forward flight (e.g. the Naval Postgraduate School MAV, Jones et al., 2005). However, it is important to emphasize that, as in nature, reversed von Karman street obtained from flapping wings is dedicated to forward flight rather than hovering flight.

In this paper, an innovative concept is investigated that can theoretically (1) allow both dynamic stall and reversed von Karman street to promote hovering flight performance while (2) avoiding zero wing velocity phases. The concept, generally referred to as the flapping-rotor (or flotor), consists in coupling both rotating and flapping motions. It was first introduced by Theodoor (2002) on a medium scale rotor. In the authors' work, the flapping motion was defined as a finite amplitude oscillating rotation of the rotor blade around an axis perpendicular to both blade and rotor axis. The flapping motion was powered while the rotating motion was induced by the flapping motion (probably through the generation of reversed von 
Karman vortex street). Since the aerodynamic performance presumably relied on flapping-to-rotating frequency ratio, their approach did not allow for any aerodynamic optimization. However, the authors pointed out that such a mechanism annihilated the rotating reaction torque, which in fact turned into a flapping reaction torque, eliminating the need for a tail rotor. Bohorquez and Pines (2002) applied the concept to a micro-rotor. The authors designed a mechanical system that allowed for both powered rotating and flapping motions. They further introduced the possibility to perform coupled pitching motion, which is defined as a finite amplitude oscillating rotation of the rotor blade around its axis. The authors were thus able to provide a preliminary evaluation of the role of the pitching-to-rotation frequency ratio on aerodynamic performance. Together with recent work by Fitchett and Chopra (2007), results suggested that flapping- and pitching-rotors can increase aerodynamic performance with respect to conventional rotors.

Despite these very preliminary results, virtually no studies provide a comprehensive analysis of flapping- and pitchingrotors. In particular, there is no clear evidence of how dynamic stall and reversed von Karman street occur along the blade span and how they are correlated with changes in aerodynamic performance. Conversely to extensively studied twodimensional cases, strong three-dimensional rotational effects may affect the vortex dynamics (Jardin and David, 2014, 2015). Such a comprehensive analysis is required to develop optimized flapping- and pitching-rotors. In this framework, the present study aims at correlating flow physics and aerodynamic performance obtained on a pitching-rotor by means of numerical simulations.

\section{Numerical setup}

The pitching-rotor consists of two untwisted rectangular blades with $4 \%$ thickness flat plate profiles. The blades have a $R=5 c$ span with a $1 c$ root cutout, where $c$ is the wing chord (see Fig. 2). The blade pitch angle is varied around the mean pitch angle $\alpha_{m}$ following a sinusoidal waveform of amplitude $\Delta \alpha$ and frequency $f_{p}: \alpha(t)=\alpha_{m}+\Delta \alpha \sin \left(2 \pi f_{p} t\right)$, where $t$ is the time. The pitch axis is located one quarter-chord from the blade leading edge. The rotating frequency $f_{r}$ leads to a maximum chord-based Reynolds number (at the blade tip) on the order of 12000.

The three-dimensional unsteady Reynolds-averaged Navier-Stokes equations (URANS) are solved under their incompressible form using StarCCM+ commercial code (CD-Adapco, 2013). An overset grid approach is used that allows each blade mesh to move following prescribed rotating and sinusoidal pitching motions within a stationary background mesh. The structured mesh consists of 4.7 million hexahedral cells $(0.9$ million for each blade mesh and 2.9 millions for background mesh) enclosed within a parallepipedic domain of width $20 R$ and height $50 R$ (see Fig. 3). The boundary conditions upstream and downstream of the rotor are implemented as pressure Dirichlet conditions while the periphery of the parallepipedic domain is defined using a slip-wall condition. The blades are modelled as non-slip surfaces. Blade mesh is moved with a time step that meets the Courant-Friedrichs-Lewy condition. Therefore, the time step is adjusted to pitching motion parameters with at least 720 time steps per rotating period. Both spatial and temporal discretizations are achieved using second-order schemes. Momentum and continuity equations are solved in an uncoupled manner using a predictor-corrector approach. Specifically, a colocated variable arrangement and a Rhie-and-Chow-type pressure-velocity coupling combined with a SIMPLE-type algorithm are used (Rhie and Chow, 1983; Ferziger and Peric, 2002). Finally, the Spalart-
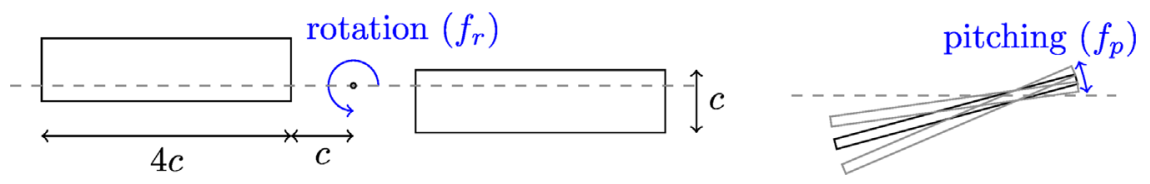

Fig. 2. Sketch of the rotor geometry and kinematics: top view (left) and side view (right).

(a)

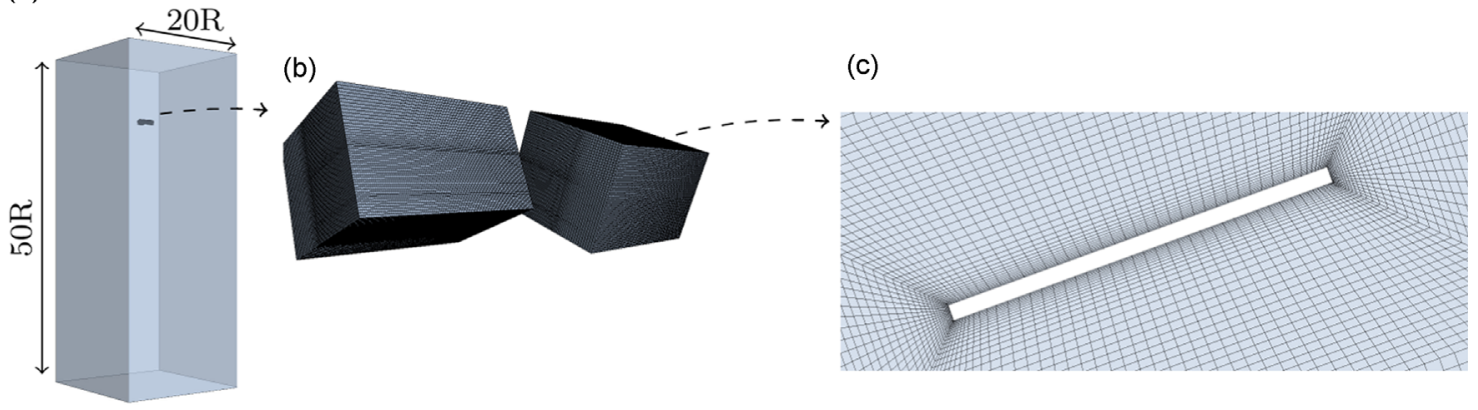

Fig. 3. Parallepipedic computational domain (a), overset blade mesh (b) and close-up view of the cross-sectional blade mesh (c). 
Allmaras model (Spalart and Allmaras, 1992) is employed for URANS turbulence closure with maximum $y^{+}$values on the order of 1 .

Convergence tests (see Appendix A) are performed for two non-pitching and two pitching cases to ensure that the results are independent to the time step and to the number of cells. Moreover, at least 9 rotation periods (and up to 16) are required for the flow to reach a periodic state.

In addition, results for non-pitching cases are compared with available experimental data obtained at a Reynolds number of 60000 (see Jardin et al., 2015 for details on the experimental approach). Fig. 4 shows that experimental data match the mean rotation torque $\overline{Q_{r}}$ versus mean thrust $\bar{T}$ curve computed using the present numerical approach within reasonable accuracy. It should further be noticed that the blade geometry (i.e. sharp leading edge) makes flow separation less sensitive to turbulence model which contributes to the robustness of the numerical approach.

\section{Investigated quantities}

Results obtained from the previously described numerical approach are analyzed in terms of aerodynamic performance and flow structure. Basically, aerodynamic performance can be quantified using mean rotor thrust $\bar{T}$ and mean rotation and pitching torques $\overline{Q_{r}}$ and $\overline{Q_{p}}$ respectively. However, deeper understanding can be gained by plotting the mean figure of merit $\overline{F M}$ versus mean thrust coefficient $\overline{C_{T}}$ map. In practice, $\overline{F M}$ and $\overline{C_{T}}$ can be viewed as measures of flight endurance and payload respectively and are therefore of paramount importance. $\overline{F M}$ is derived from $\overline{C_{T}}$ and $\overline{C_{P}}$ :

$$
\overline{F M}=\frac{{\overline{C_{T}}}^{3 / 2}}{\sqrt{2 C_{P}}},
$$

where

$$
\begin{aligned}
& C_{T}=\frac{T}{\rho\left(\pi R^{2}\right)\left(\Omega_{r} R\right)^{2}}, \\
& C_{P}=\frac{P}{\rho\left(\pi R^{2}\right)\left(\Omega_{r} R\right)^{3}},
\end{aligned}
$$

and

$$
P=\Omega_{r} Q_{r}+\Omega_{p} Q_{p}
$$

Note that the rotation speed $\Omega_{r}$ is constant, and thus equals $2 \pi f_{r}$, while the pitching speed $\Omega_{p}$ is not. In addition, we introduce $\overline{C_{P_{r}}}$ and $\overline{F M_{r}}$ where $\Omega_{p} Q_{p}$ is neglected in Eq. (4). As a complement, we introduce $\overline{C_{P_{p}}}$ where only $\Omega_{p} Q_{p}$ is considered in Eq. (4).

$T, Q_{r}$ and $Q_{p}$ are calculated by spatially integrating pressure and viscous forces on both blades at each time step. Their mean values result from the temporal integration of instantaneous values over one rotation period once the flow has reached a periodic state.

Aerodynamic performance is then correlated with $\lambda_{2}$-criterion isolines (Jeong and Hussain, 1995), as well as spanwise vorticity and tangential velocity contours.

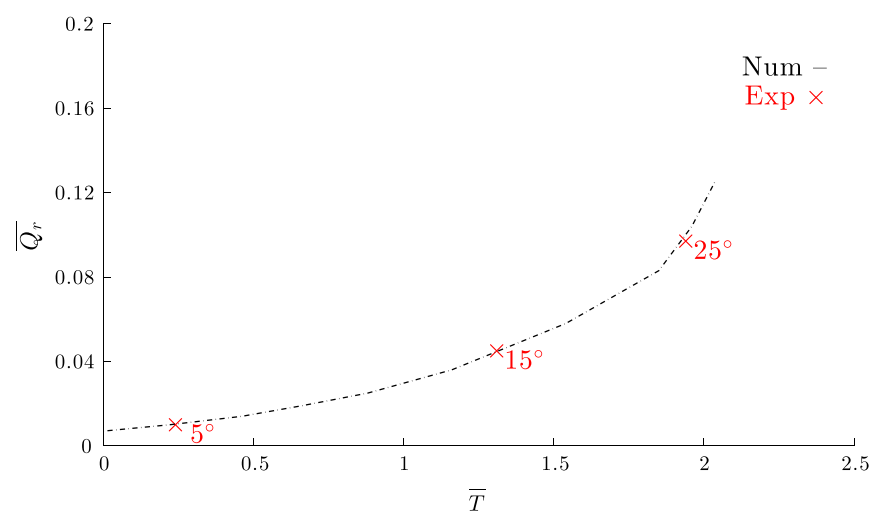

Fig. 4. $\overline{Q_{r}}$ versus $\bar{T}$ map obtained numerically and experimentally for non-pitching cases at a Reynolds number of 60000 (blade pitch angle $\alpha_{m}$ is indicated on experimental data). 


\section{Results}

\subsection{Overall analysis of aerodynamic performance}

The $\left(\alpha_{m}, \Delta \alpha, f_{p} / f_{r}\right)$ parameter space is explored by computing 74 cases. Fig. 5 shows the $\overline{F_{M}}$ versus $\overline{C_{T}}$ map obtained from such an exploration, where $\alpha_{m} \in\left[0^{\circ} ; 40^{\circ}\right], \Delta \alpha \in\left[0^{\circ} ; 15^{\circ}\right]$ and $f_{p} / f_{r} \in[0 ; 36]$. Results are mapped using numeral symbols which indicate the pitching-to-rotation frequency ratio $f_{p} / f_{r}$. 0 values are used for non-pitching cases and therefore represents the baseline $\overline{F M}$ versus $\overline{C_{T}}$ curve. Straight dashed-lines are drawn to show the trend associated with increasing pitching-torotation frequency ratio within a given $\left(\alpha_{m}, \Delta \alpha\right)$ family. Fig. 5 provides a general picture of the results and thus offers the opportunity to reveal striking and generic features of pitching rotor aerodynamics. As such, it highlights specific configurations which will then be further investigated to understand how the flow physics is correlated with changes in aerodynamic performance.

A first striking feature is that significant gain in $\overline{F M_{r}}$ can be obtained by low amplitude $\Delta \alpha$ and high frequency $f_{p}$ pitching motions at low to moderate pitch angles $\alpha_{m}$. Typical examples of this increase include cases where $\Delta \alpha=5^{\circ}$ and where $f_{p} / f_{r}$ roughly exceeds 8 for $\alpha_{m}=5^{\circ}, 10^{\circ}$ and $15^{\circ}$ (see three leftmost dashed blue lines). Comparison within these three $\left(\alpha_{m}=5^{\circ}, \Delta \alpha=5^{\circ}\right),\left(\alpha_{m}=10^{\circ}, \Delta \alpha=5^{\circ}\right)$ and $\left(\alpha_{m}=15^{\circ}, \Delta \alpha=5^{\circ}\right)$ families highlights a clockwise tilting of the trend-lines with increasing $\alpha_{m}$. This indicates that the gain in $\overline{F M_{r}}$ progressively turns into a gain in $\overline{C_{T}}$ with increasing $\alpha_{m}$. Such an observation is confirmed at higher pitch angles where $\left(\alpha_{m}=30^{\circ}, \Delta \alpha=5^{\circ}\right)$ and $\left(\alpha_{m}=40^{\circ}, \Delta \alpha=5^{\circ}\right)$ trend lines are nearly horizontal (two rightmost dashed blue lines). Note however that the gain in $\overline{C_{T}}$ at high pitch angles is not significant.

Second, the increase in $\overline{F M_{r}}$ observed at moderate $\alpha_{m}$ vanishes at higher values of $\Delta \alpha$. In particular, $\left(\alpha_{m}=10^{\circ}, \Delta \alpha=10^{\circ}\right)$, $\left(\alpha_{m}=15^{\circ}, \Delta \alpha=10^{\circ}\right)$ and $\left(\alpha_{m}=15^{\circ}, \Delta \alpha=15^{\circ}\right)$ families exhibit trend lines that lie below the baseline curve, indicating a detrimental effect of pitching motion on aerodynamic performance. On the other hand, it can be seen that higher values of $\Delta \alpha$ enhance $\overline{C_{T}}$ at high $\alpha_{m}$ and moderate $f_{p} / f_{r}$. A good example of such a lift-enhancement is given by the ( $\alpha_{m}=40^{\circ}, \Delta \alpha=15^{\circ}, f_{p} / f_{r}=5$ ) case (rightmost dotted red line).

Therefore, the simple analysis of the $\overline{F M_{r}}$ versus $\overline{C_{T}}$ map suggests that relevant pitching motions can be treated following two distinct groups:

(A) Low to moderate pitch angles $\alpha_{m}$ (e.g. $5^{\circ}, 10^{\circ}$, and $15^{\circ}$ ), low pitching amplitudes $\Delta \alpha\left(<5^{\circ}\right)$ and high pitching-torotation frequency ratio $f_{p} / f_{r}(>8)$.

(B) High pitch angles $\alpha_{m}$ (e.g. $40^{\circ}$ ), moderate pitching amplitudes $\Delta \alpha$ (e.g. $15^{\circ}$ ) and moderate pitching-to-rotation frequency ratio $f_{p} / f_{r}$ (e.g. 5).

These groups, hereafter referred to as group A and B respectively, are believed to be related to distinct flow features. Prior to analyzing the flow structure, the $\overline{F M}$ versus $\overline{C_{T}}$ map displayed in Fig. 6 can provide further evidence of such a statement.

Interestingly, cases with low $\Delta \alpha$ and high $f_{p} / f_{r}$ at low to moderate $\alpha_{m}$ (group A) lead to a drastic reduction in $\overline{F M}$. In light of the aforementioned results, this suggests that the rotation torque partly turns into a pitching torque through an inefficient process. In other words, the blades rotate through both powered rotation and pitch-induced rotation but pitchinduced rotation is much less efficient than powered rotation.

Conversely, cases with moderate $\Delta \alpha$ and moderate $f_{p} / f_{r}$ at high $\alpha_{m}$ (group B) do not lead to a significant drop in $\overline{F M}$. Therefore, there is no clear evidence of pitch-induced rotation and the blades mainly rotate through powered rotation. This demonstrates that pitching motions can increase aerodynamic lift without global efficiency penalties.

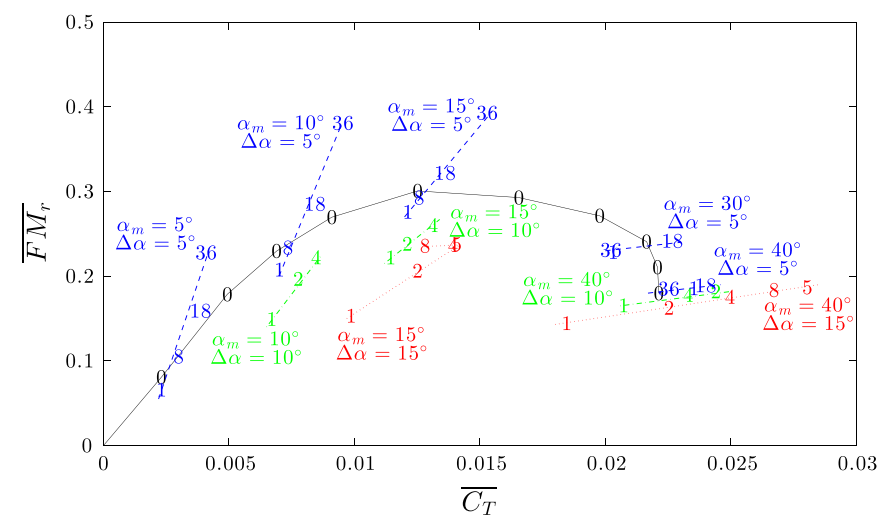

Fig. 5. $\overline{F M_{r}}$ versus $\overline{C_{T}}$ map obtained for non-pitching and pitching cases. Numeral symbols indicate the pitching-to-rotation frequency. Dashed blue, dashdotted green and dotted red lines show the trend associated with increasing pitching-to-rotation frequency ratio within a given $\left(\alpha_{m}, \Delta \alpha\right)$ family, with $\Delta \alpha=5^{\circ}, 10^{\circ}$ and $15^{\circ}$ respectively. (For interpretation of the references to color in this figure caption, the reader is referred to the web version of this paper.) 
Overall, conclusions drawn from Fig. 6 strengthen the idea that distinct flow phenomena are involved at low $\Delta \alpha$, high $f_{p} / f_{r}$, low to moderate $\alpha_{m}$ (group A) and moderate $\Delta \alpha$, moderate $f_{p} / f_{r}$, high $\alpha_{m}$ (group B). Next section aims at correlating such phenomena to aerodynamic performance.

\subsection{Correlation between flow structure and aerodynamic performance}

\subsubsection{Baseline}

Fig. 7 depicts the $\overline{F M}$ versus $\overline{C_{T}}$ map obtained for the baseline cases (i.e. no pitching motion), together with instantaneous spanwise vorticity contours and $\lambda_{2}$-isolines in three spanwise cross sections located $2 c, 3 c$ and $4 c$ away from the rotor center. Instantaneous snapshots are shown for an arbitrary instant and are not representative of the mean or phase-averaged flow when the flow is unsteady. In addition, blue dots indicate instantaneous values of $F M$ and $C_{T}$. It is shown that $\overline{F M}$ and $\overline{C_{T}}$ increases with $\alpha_{m}$ up to an optimal rotor efficiency at $\alpha_{m} \approx 15^{\circ}$. At low $\alpha_{m}$ (e.g. $5^{\circ}$ ), the flow is mostly attached, yet with small recirculation regions immediately after the sharp leading edge. As $\alpha_{m}$ is increased (e.g. $15^{\circ}$ ), the flow tends to massively separate from the leading edge, forming a conical leading edge vortex that remains robustly attached to the upper surface of the blade. By virtually increasing the blade profile camber, such a LEV contributes to increasing both $\overline{F M}$ and $\overline{C_{T}}$ up to the optimal $\alpha_{m} \approx 15^{\circ}$ value. As $\alpha_{m}$ is further increased beyond $15^{\circ}$, the LEV becomes unstable in the outboard region of the blade (i.e. the outboard region stalls) while remaining stable in the inboard region. Note that the development of stable LEVs is a ubiquitous feature observed in nature and was recently found to be promoted by the relative importance of Coriolis effects (Lentink and Dickinson, 2009; Jardin and David, 2014, 2015), in a region typically extending from the rotor center to approximately $4 c$ away from it (Kruyt et al., 2015). Concomitant occurrence of outboard stall and inboard LEV growth with $\alpha_{m}$ leads to a decrease in $\overline{F M}$ and an increase in $\overline{C_{T}}$ beyond $\alpha_{m}=15^{\circ}$. In addition, unstable flow on the outboard region of the blade generates loads fluctuations. Blue dots in Fig. 7 indicate that these fluctuations become significant near $\alpha_{m} \approx 25^{\circ}$. As $\alpha_{m}$

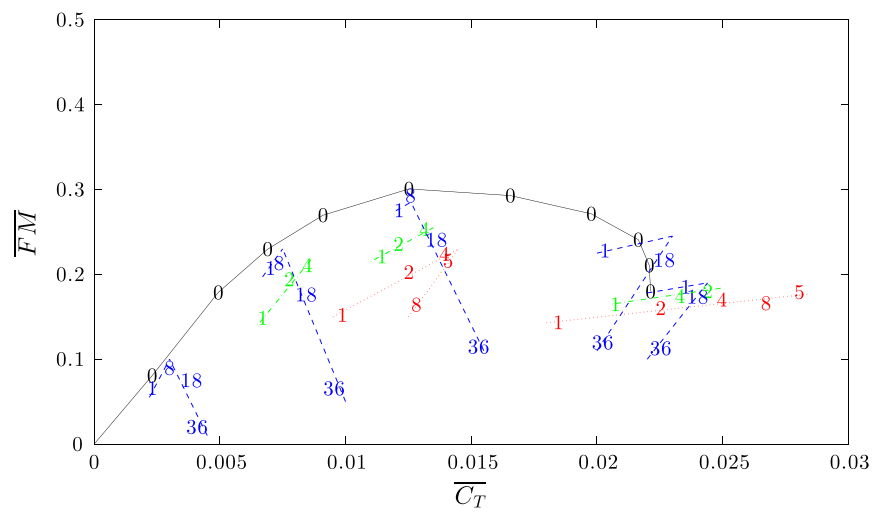

Fig. 6. $\overline{F M}$ versus $\overline{C_{T}}$ map obtained for non-pitching and pitching cases. Numeral symbols indicate the pitching-to-rotation frequency. Dashed blue, dashdotted green and dotted red lines show the trend associated with increasing pitching-to-rotation frequency ratio within a given $\left(\alpha_{m}, \Delta \alpha\right)$ family. (For interpretation of the references to color in this figure caption, the reader is referred to the web version of this paper.)

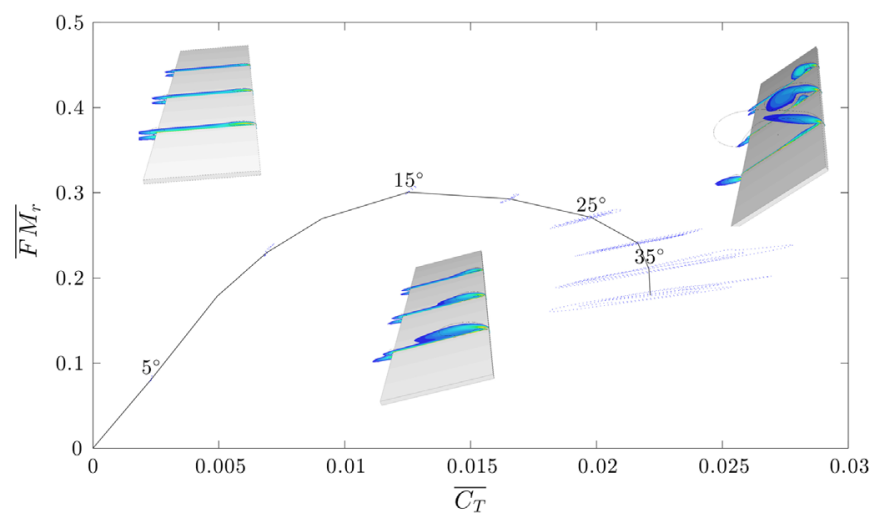

Fig. 7. $\overline{F M}$ versus $\overline{C_{T}}$ map obtained for non-pitching cases. Vorticity flow fields in three spanwise cross sections are shown for three relevant values of $\alpha_{m}=5^{\circ}, 15^{\circ}$ and $35^{\circ}$. Blue dots indicate instantaneous values of $F M$ and $C_{T}$. (For interpretation of the references to color in this figure caption, the reader is referred to the web version of this paper.) 
is further increased, unsteady separation on the outboard region of the blade progressively contaminates the inboard region, leading to a significant increase in loads fluctuations (e.g. $35^{\circ}$ ), until the whole blade stalls.

\subsubsection{Group A}

In this section, we focus on cases that promote an increase in $\overline{F M_{r}}$. In particular, we look at the evolution of $\overline{C_{T}}, \overline{C_{P_{r}}}, \overline{F M_{r}}$, $\overline{C_{P_{p}}}$ and $\overline{F M}$ as a function of $f_{p} / f_{r}$ for $\left(\alpha_{m}=5^{\circ}, \Delta \alpha=5^{\circ}\right),\left(\alpha_{m}=10^{\circ}, \Delta \alpha=5^{\circ}\right)$ and $\left(\alpha_{m}=15^{\circ}, \Delta \alpha=5^{\circ}\right)$ families $($ Fig. 8$)$.

First, dash-dotted trend lines in Fig. $8\left(\right.$ a) indicate that $\overline{C_{T}}$ roughly increases linearly with $f_{p} / f_{r}$. These trend lines lie above the corresponding horizontal dotted lines which depict $\overline{C_{T}}$ values obtained without pitching at $\alpha_{m}=5^{\circ}, \alpha_{m}=10^{\circ}$ and $\alpha_{m}=15^{\circ}$. This shows that at a given $\alpha_{m}$, imposing a low-amplitude $\left(\Delta \alpha=5^{\circ}\right.$ ) pitching motion increases $\overline{C_{T}}$.

Second, dash-dotted trend lines in Fig. 8(b) indicate that $\overline{C_{P_{r}}}$ is not significantly affected by $f_{p} / f_{r}$, although we do note a slight decrease with increasing $f_{p} / f_{r}$. In addition, $\overline{C_{P_{r}}}$ values of low-amplitude $\left(\Delta \alpha=5^{\circ}\right)$ pitching cases lie around the $\overline{C_{P_{r}}}$ values obtained for non-pitching cases.

As a consequence, $\overline{F M_{r}}$ increases linearly with $f_{p} / f_{r}$ such that $\overline{F M_{r}}$ dash-dotted trend lines cross horizontal dashed lines at some points located in a region roughly located between $f_{p} / f_{r}=4$ and $f_{p} / f_{r}=9$. This region is displayed using light gray color in Fig. 8(c).

Finally, Fig. 8(d) indicates that $\overline{C_{P_{p}}}$ grows exponentially with $f_{p} / f_{r}$. Here, it can also be pointed out that $\overline{C_{P_{p}}}$ does not depend on $\alpha_{m}$ and is mostly influenced by pitching accelerations (i.e. driven by pitching amplitude and frequency) and associated added mass effects.
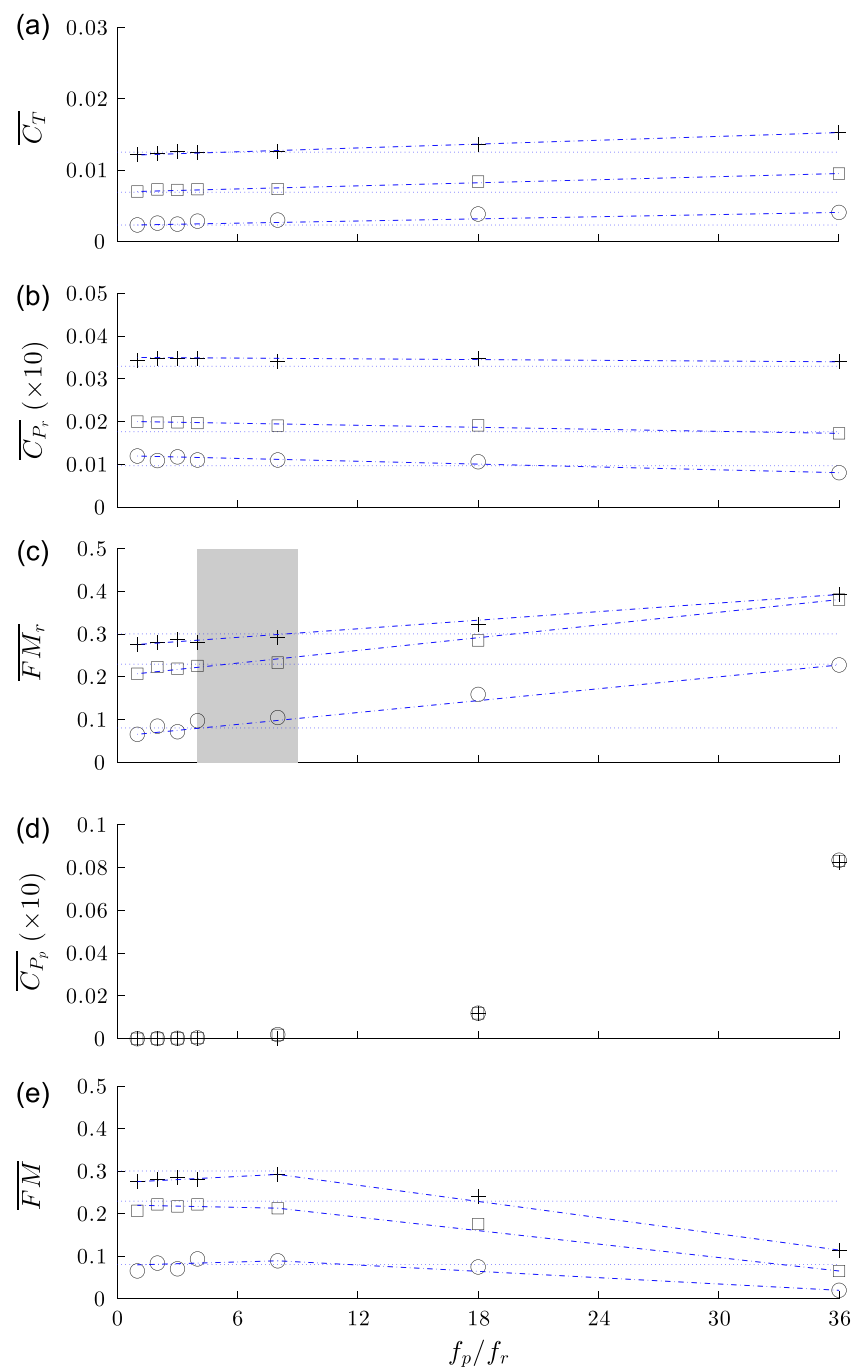

Fig. 8. $\overline{C_{T}}$ (a), $\overline{C_{P_{r}}}$ (b), $\overline{F M_{r}}$ (c), $\overline{C_{P_{p}}}$ (d) and $\overline{F M}$ (e) as a function of $f_{p} / f_{r}$ for $\Delta \alpha=5^{\circ}$ and $\alpha_{m}=5^{\circ}(\circ), \alpha_{m}=10^{\circ}(\square), \alpha_{m}=15^{\circ}(+)$. Dashed lines show the trend associated with increasing pitching-to-rotation frequency ratio within a given $\left(\alpha_{m}, \Delta \alpha\right)$ family. Dotted lines indicate values obtained for corresponding static cases. 
All these observations explain the double-trend evolution of $\overline{F M}$ with $f_{p} / f_{r}$ displayed in Fig. 8(e). Interestingly, changes in the evolution of $\overline{F M}$ with $f_{p} / f_{r}$ (roughly constant trend at low $f_{p} / f_{r}$ and decreasing at higher $f_{p} / f_{r}$ ) also occur in the lightgray region.

In light of these results, we ask if this light-gray region indicates a transition between specific flow phenomena occurring at low and high $f_{p} / f_{r}$ respectively? To answer this question, we analyze the flow in terms of vorticity and $\lambda_{2}$-criterion for two extreme cases $f_{p} / f_{r}=1$ and $f_{p} / f_{r}=18$, with $\alpha_{m}=10^{\circ}$ and $\Delta \alpha=5^{\circ}$. Fig. 9 displays instantaneous snapshots of spanwise vorticity contours and $\lambda_{2}$ isolines in three spanwise cross sections located $2,3 c$ and $4 c$ away from the rotor center, at four instants $N / 4 f_{p}$. Because the flow exhibits strong periodicity (see Appendix A), instantaneous snapshots are here representative of phase-averaged flow behavior.

The $f_{p} / f_{r}=1$ case is displayed in the top row. Overall, it can be observed that the flow is driven by the development of a conical LEV close to the upper surface of the blade. The scenario over a pitching period is very simple:

(a) The LEV reaches its largest size at $t=0 / 4 f_{p}$ where the instantaneous pitch angle is maximum $\left(\alpha=15^{\circ}\right)$, hence producing the largest instantaneous thrust.

(b) It then decreases in size as the pitch angle decreases down to $\alpha=10^{\circ}$ at $t=1 / 4 f_{p}$.

(c) And nearly vanishes at $t=2 / 4 f_{p}$ where the pitch angle is minimum $\left(\alpha=5^{\circ}\right)$, hence producing the lowest instantaneous thrust.

(d) The LEV develops again as the pitch angle increases up to $\alpha=10^{\circ}$ at $t=3 / 4 f_{p}$ and $\alpha=15^{\circ}$ at $t=4 / 4 f_{p}$, which loops the pitching cycle.

Here, the flow structure at $t=0 / 4 f_{p}, t=1 / 4 f_{p}$ and $t=2 / 4 f_{p}$ is very similar to that observed for the static cases $f_{p} / f_{r}=0$ at $\alpha_{m}=15^{\circ}, \alpha_{m}=10^{\circ}$ and $\alpha_{m}=5^{\circ}$ respectively. In other words, the flow can be considered as quasi-steady and the mean thrust and torque coefficients do not fundamentally differ from those obtained for the static case $f_{p} / f_{r}=0$ at $\alpha_{m}=10^{\circ}$. Yet, we do note a slight difference between the LEV at $t=1 / 4 f_{p}$ and that at $t=3 / 4 f_{p}$ (where the instantaneous pitch angles are similar), which indicates hysteresis effects between nose-down and nose-up pitching phases.

The $f_{p} / f_{r}=18$ case shown in the bottom row exhibits a more complex scenario:

(e) The inception of the LEV can be observed at $t=0 / 4 f_{p}$ where the instantaneous pitch angle is maximum $\left(\alpha=15^{\circ}\right)$.

(f) The LEV grows in size and rapidly sheds from the leading edge as the instantaneous pitch angle decreases $\left(\alpha=10^{\circ}\right.$ at $\left.t=1 / 4 f_{p}\right)$.

(g) Once shed, the LEV 'rolls' on the upper surface of the blade towards the trailing edge $\left(\alpha=5^{\circ}\right.$ at $\left.t=2 / 4 f_{p}\right)$.

(h) The LEV has not yet reached the trailing edge as the pitch angle increases again to $\alpha=10^{\circ}$ at $t=3 / 4 f_{p}$.

Here, conversely to the $f_{p} / f_{r}=1$ case, the LEV appears as a prominent flow feature not only at maximum pitch angle $\left(t=0 / 4 f_{p}\right)$, but also at minimum pitch angle $\left(t=2 / 4 f_{p}\right)$. The main outcome of such vortex dynamics is the increase in circulatory forces (i.e. vortex-induced forces, as opposed to non-circulatory forces or added mass) at low instantaneous pitch angles, with respect to quasi-steady circulatory forces. Therefore, the mean thrust $\overline{C_{T}}$ is increased with respect to that obtained for the static case $f_{p} / f_{r}=0$ at $\alpha_{m}=10^{\circ}$. Such an increase, revealed in Fig. 8(a), becomes significant if the pitching-
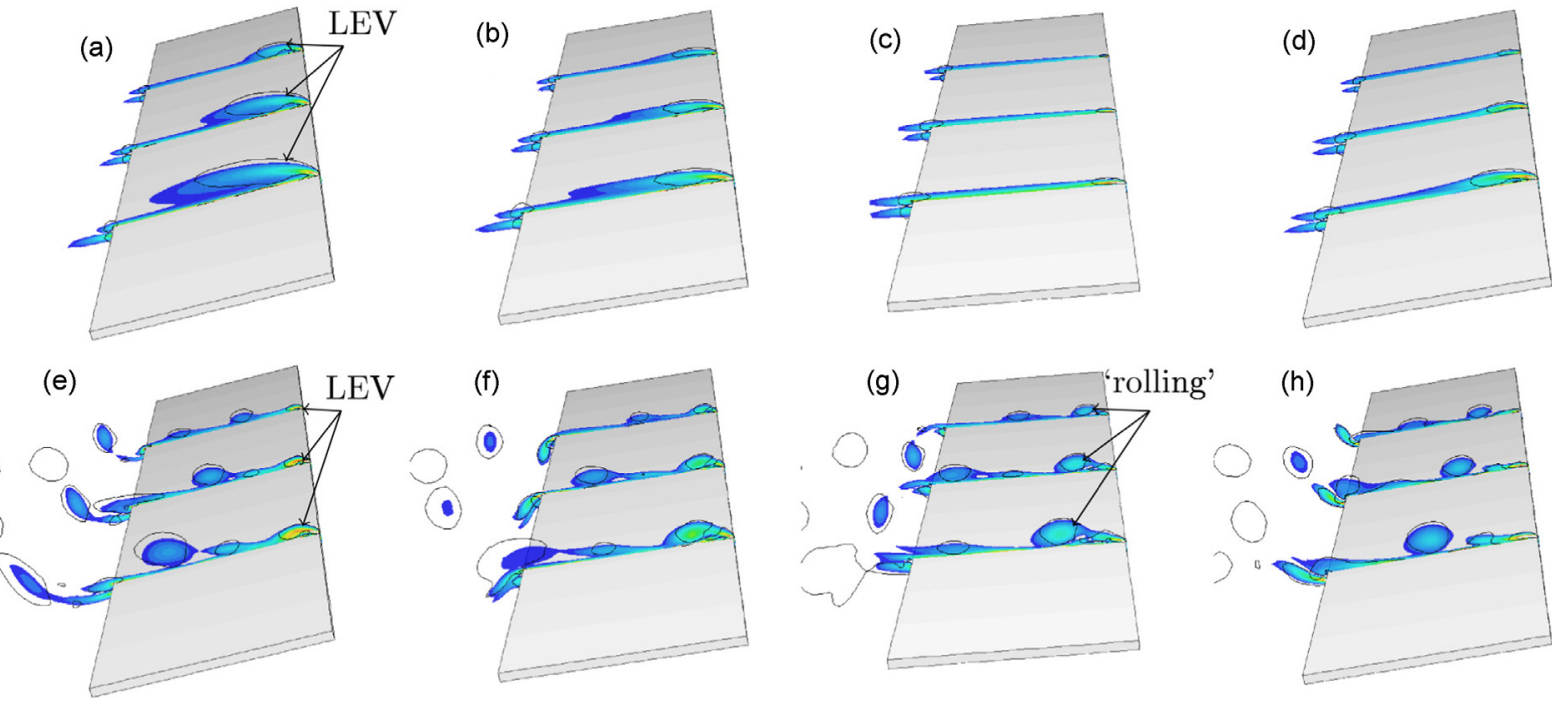

Fig. 9. Cross-sectional spanwise vorticity contours and $\lambda_{2}$-criterion isolines obtained for $f_{p} / f_{r}=1$ (a)-(d) and $f_{p} / f_{r}=18(\mathrm{e})-(\mathrm{h})$ at times $0 / 4 f_{p}(\mathrm{a})$ and $(\mathrm{e})$, $1 / 4 f_{p}$ (b) and (f), $2 / 4 f_{p}$ (e) and (g) and $3 / 4 f_{p}(\mathrm{~d})$ and (h). For both cases $\alpha_{m}=10^{\circ}$ and $\Delta \alpha=5^{\circ}$. 


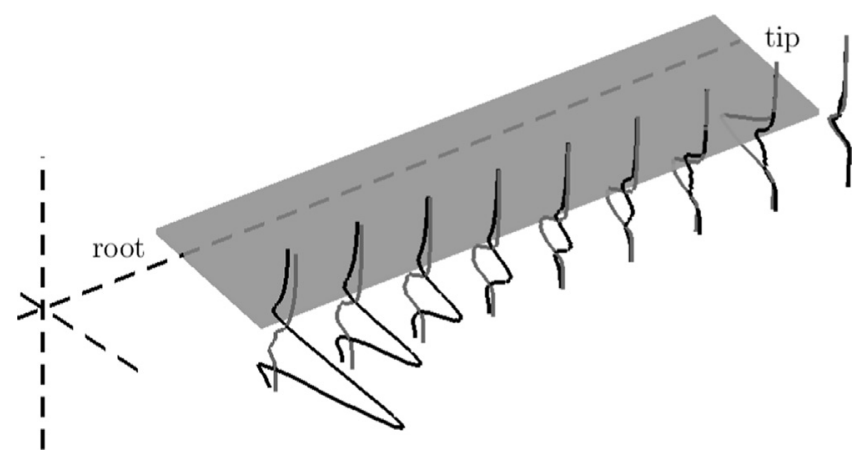

Fig. 10. Mean tangential velocity profiles obtained one chord downstream of the blade pitching axis for $f_{p} / f_{r}=1$ (gray) and $f_{p} / f_{r}=18$ (black). For both cases $\alpha_{m}=10^{\circ}$ and $\Delta \alpha=5^{\circ}$.

to-rotation frequency ratio $f_{p} / f_{r}$ is sufficiently high to ensure that the LEV benefits to low pitch angle phases, i.e. the LEV does not reach the trailing edge and does not shed into the wake before a new LEV is formed. Furthermore, all these observations suggest that the mean thrust increases with the number of LEV formed during a pitching period as $f_{p} / f_{r}$ increases.

On the other hand, an increase in circulatory force should also lead to an increase in rotation torque, which is not in line with conclusions drawn from Fig. 8(b). Two reasons can be invoked to clarify this point. First, it is interesting to note that 'extra' circulatory forces induced by the LEV at low instantaneous pitch angle contribute more to thrust than torque, precisely because of the low pitch angle (projection of the circulatory force in the rotor disk is small). Second, the most relevant mechanism here is associated with a rearrangement of the shed vortices in the wake of the blade due to increased pitching frequency (as previously illustrated in Fig. 1). While a strong velocity deficit is observed in the wake of the $f_{p} / f_{r}=1$ case, which is indicative of rotation torque, such a velocity deficit is significantly reduced in the $f_{p} / f_{r}=18$ case, and even turns into a velocity surplus in the inboard region of the blade, which is indicative of local reversed von Karman street and local pitch-induced rotation (Fig. 10). This explains why the mean rotation torque $C_{P_{r}}$ does not increase with increased circulatory forces at high $f_{p} / f_{r}$, and even slightly decreases at some point.

Overall, it can be concluded that the gain in $\overline{F M_{r}}$ is related to both LEV's development and 'surface-rolling', and TEVs and LEVs rearrangement in the wake (potentially leading to reversed von Karman street). The occurrence of such mechanisms depend on the ratio between the time of advection of the LEV and the pitching frequency, which can be quantified by the reduced pitching frequency $c f_{p} / r f_{r}$, where $r$ is the local blade radius. Since the time of advection of the LEV varies along the blade span due to non-uniform oncoming flow at each blade section, these mechanisms first appear at the blade root (i.e. small $r$ values) at low $f_{p} / f_{r}$ values and then propagate towards the blade tip as $f_{p} / f_{r}$ is increased. This makes the transition between low and high $f_{p} / f_{r}$ regimes inherently smooth. In addition, although spanwise varying oncoming flow along the blade span tends to smoothen this transition, recent work by Mackowski and Williamson (2015) suggested that the evolution of force production with pitching frequencies on two-dimensional pitching airfoils follows a relatively smooth trend. Finally, it can be seen that velocity surplus in the wake of the blade occurs at local radii below $3.5 c$, hence local reduced frequencies above 5, which is in line with two-dimensional studies (Sarkar and Venkatraman, 2006). This suggests that, in these specific cases, the flow can reasonably be considered as quasi-two-dimensional, in the sense that the flow behaves locally (at a given radial position) as a two-dimensional one.

\subsubsection{Group B}

In this section, we focus on cases that promote an increase in $\overline{C_{T}}$. In particular, we look at the evolution of $\overline{C_{T}}, \overline{C_{P_{r}}}, \overline{F M_{r}}$, $\overline{C_{P_{p}}}$ and $\overline{F M}$ as a function of $f_{p} / f_{r}$ for $\left(\alpha_{m}=40^{\circ}, \Delta \alpha=5^{\circ}\right),\left(\alpha_{m}=40^{\circ}, \Delta \alpha=10^{\circ}\right)$ and $\left(\alpha_{m}=40^{\circ}, \Delta \alpha=15^{\circ}\right)$ families (Fig. 11).

On the contrary to group $\mathrm{A}$, it can be seen from Fig. 11(a) that the evolution of $\overline{C_{T}}$ with $f_{p} / f_{r}$ exhibits a highly non-linear trend. This suggests that for these specific cases, the flow is driven by massive separation leading to highly unsteady flow features. Therefore, it clearly appears that low $f_{p} / f_{r}$ can be detrimental to thrust production with respect to the static case $f_{p} / f_{r}=0$ at $\alpha_{m}=40^{\circ}$ whereas a gain in $\overline{C_{T}}$ can be expected at higher $f_{p} / f_{r}(>4)$.

The evolution of $\overline{C_{P_{r}}}$ with $f_{p} / f_{r}$ shown in Fig. 11(b) exhibits a rather similar trend to that observed for $\overline{C_{T}}$. As a result, $\overline{F M_{r}}$ is not significantly affected with respect to that obtained for the static case (Fig. 11(c)).

On the other hand, increasing $f_{p} / f_{r}$ tends to significantly increase $\overline{C_{P_{p}}}$ (Fig. 11(d)). As previously mentioned, added mass effects play a major role in $\overline{C_{P_{p}}}$ generation. This is further highlighted here by the difference observed in $\overline{C_{P_{p}}}$ for distinct $\Delta \alpha$ cases, added mass effects being increased with increasing $\Delta \alpha$ at a given $f_{p} / f_{r}$ value.

However, $\overline{C_{P_{p}}}$ levels are an order of magnitude lower than $\overline{C_{P_{r}}}$ levels such that the global efficiency $\overline{F M}$ is not drastically altered with respect to that of the static case (Fig. 11(e)).

In order to gain further insight into the thrust producing mechanism, we analyze the flow in terms of vorticity and $\lambda_{2}$ criterion for two extreme cases $f_{p} / f_{r}=1$ and $f_{p} / f_{r}=5$, with $\alpha_{m}=40^{\circ}$ and $\Delta \alpha=15^{\circ}$. Fig. 12 displays instantaneous snapshots of spanwise vorticity contours and $\lambda_{2}$ isolines in three spanwise cross sections located $2,3 c$ and $4 c$ away from the rotor 

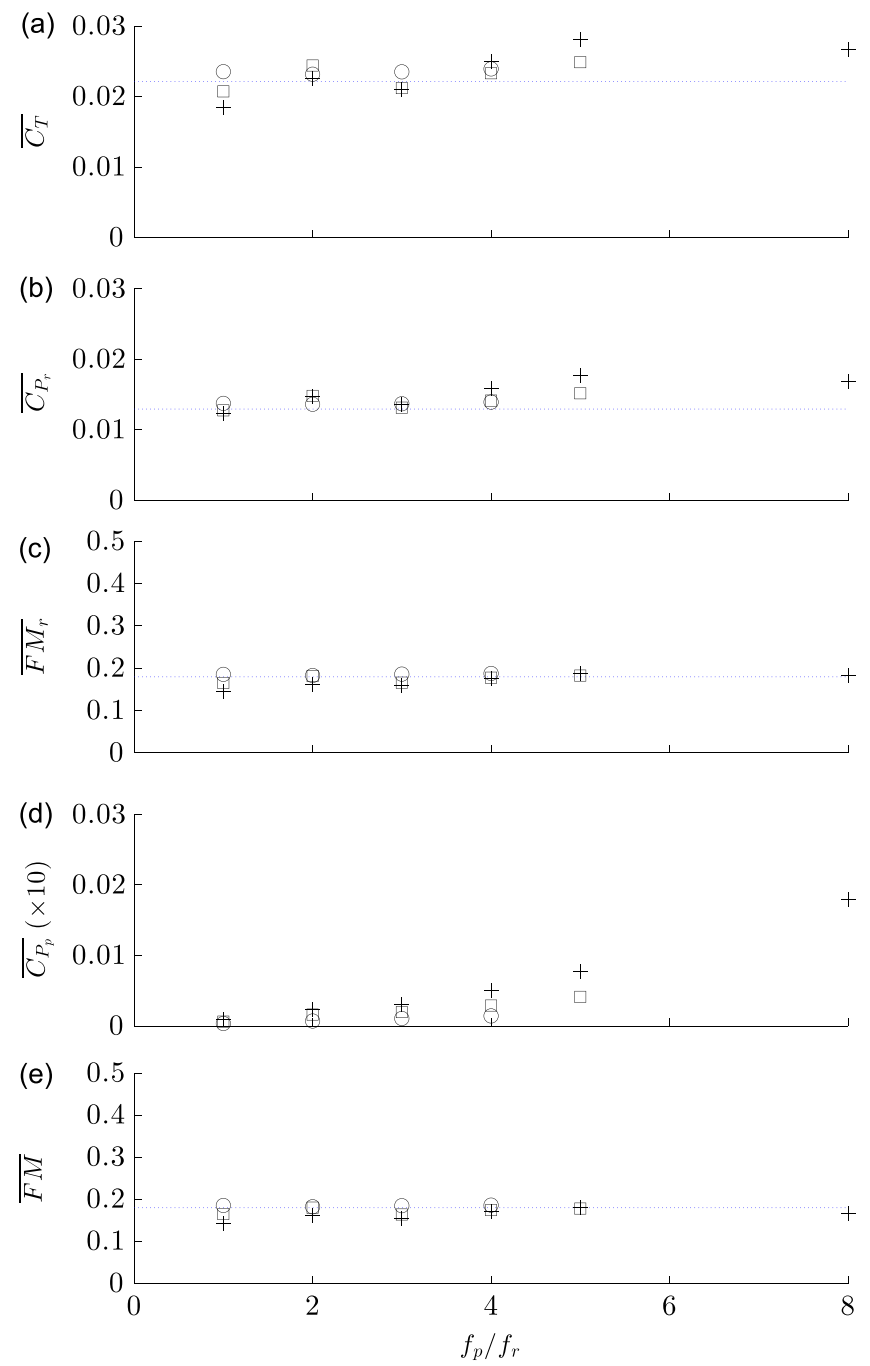

Fig. 11. $\overline{C_{T}}$ (a), $\overline{C_{P_{r}}}$ (b), $\overline{F M_{r}}$ (c), $\overline{C_{P_{p}}}$ (d) and $\overline{F M}$ (e) as a function of $f_{p} / f_{r}$ for $\alpha_{m}=40^{\circ}$ and $\Delta \alpha=5^{\circ}(\circ), \Delta \alpha=10^{\circ}(\square), \Delta \alpha=15^{\circ}(+)$. Dotted lines indicate values obtained for corresponding static cases.

center, at four instants $N / 4 f_{p}$. Here again, instantaneous snapshots are believed to be representative of phase-averaged flow behavior due to flow periodicity (see Appendix A).

The $f_{p} / f_{r}=1$ case is displayed in the top row. The scenario over a pitching period is as follows:

(a) At $t=0 / 4 f_{p}$, the instantaneous pitch angle is maximum $\left(\alpha=55^{\circ}\right)$; most of the blade span is stalled and is characterized by the shedding of both LEVs and TEVs.

(b) The flow remains mostly unstable along the blade span as $\alpha$ reduces to $40^{\circ}$ at $t=1 / 4 f_{p}$, with clear evidence of vortex shedding.

(c) The flow progressively reattaches to the blade as $\alpha$ is further reduced to $25^{\circ}$ at $t=2 / 4 f_{p}$, showing evidence of a stable LEV along most of the blade span, although the LEV still tends to detach from the outboard region of the blade.

(d) The LEV in the inboard region grows again as $\alpha$ is increased to $40^{\circ}$ at $t=3 / 4 f_{p}$ and again becomes highly unstable in the outboard region.

Here, phases at very high pitch angles induce an unstable and highly unsteady flow along most of the wing span. In addition, characteristic frequencies associated with this unsteadiness (i.e. LEVs and TEVs shedding) are strongly coupled with the pitching frequency such that the $f_{p} / f_{r}=1$ case cannot be treated as a quasi-steady case. This is corroborated by non-negligible differences between flow structure observed at $t=1 / 4 f_{p}$ and $t=3 / 4 f_{p}$ (where the instantaneous pitch angles are similar), which highlight hysteresis effects between nose-down and nose-up pitching phases.

The $f_{p} / f_{r}=5$ case depicted in the bottom row demonstrates a completely different scenario: 
(a)

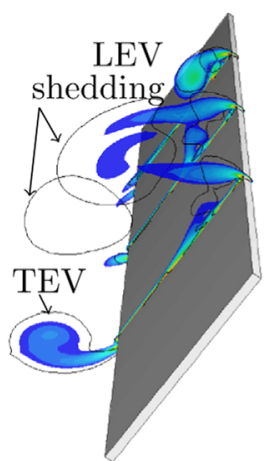

(e)

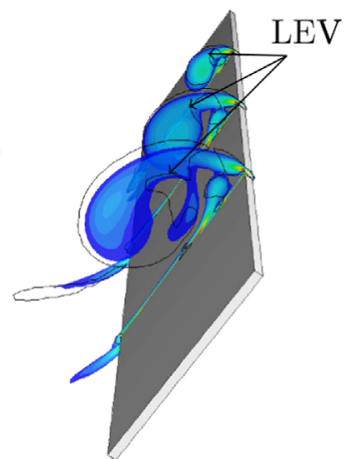

(b)

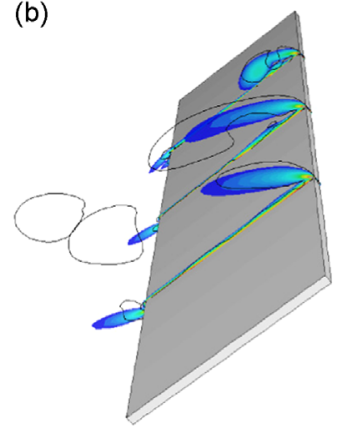

(f)

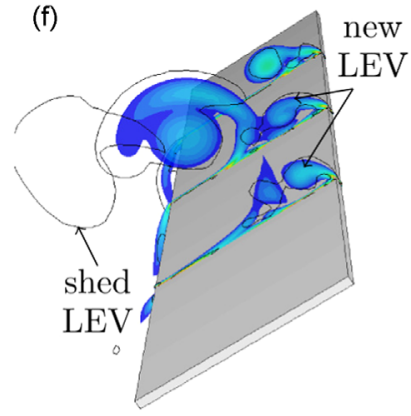

(c)
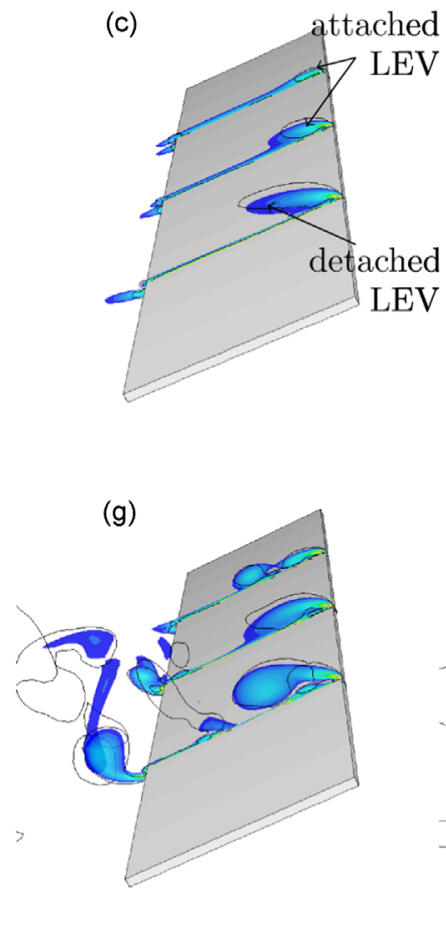

(d)

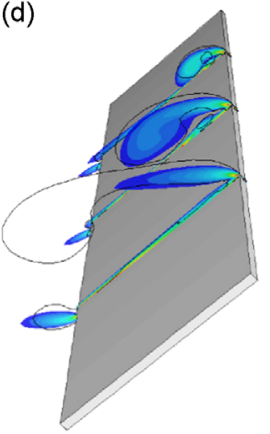

(h)

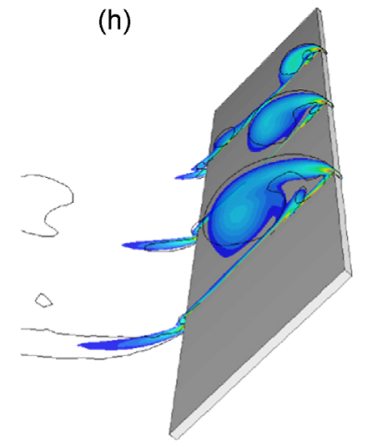

Fig. 12. Cross-sectional spanwise vorticity contours and $\lambda_{2}$-criterion isolines obtained for $f_{p} / f_{r}=1$ (a)-(d) and $f_{p} / f_{r}=5(\mathrm{e})-(\mathrm{h})$ at times $0 / 4 f_{p}(\mathrm{a})$ and (e), $1 / 4 f_{p}$ (b) and (f), $2 / 4 f_{p}(\mathrm{e})$ and (g) and $3 / 4 f_{p}(\mathrm{~d})$ and (h). For both cases $\alpha_{m}=40^{\circ}$ and $\Delta \alpha=15^{\circ}$.

(a) At $t=0 / 4 f_{p}$, the instantaneous pitch angle is maximum $\left(\alpha=55^{\circ}\right)$ and the LEV reaches its largest size.

(b) The LEV sheds into the wake in the outboard region of the blade as $\alpha$ decreases to $40^{\circ}$ at $t=1 / 4 f_{p}$, and is advected close to the upper surface in the mid-blade section, leaving room for a new LEV to develop.

(c) At $t=2 / 4 f_{p}$ where the instantaneous pitch angle is minimum $\left(\alpha=25^{\circ}\right)$, a new LEV develops.

(d) That remains close to the upper surface of the blade as $\alpha$ increases back to $\alpha=40^{\circ}$ at $t=3 / 4 f_{p}$.

Here, the higher pitching frequency helps take advantage of dynamic stall process by allowing for a strong LEV to be formed at high instantaneous pitch angle $\left(t=0 / 4 f_{p}\right)$ and starting nose-down pitching before the LEV sheds into the wake. In addition, a mechanism rather similar to that observed for the $\left(\alpha_{m}=10^{\circ}, \Delta \alpha=5^{\circ}, f_{p} / f_{r}=18\right)$ case arises in that the LEV generated at maximum instantaneous pitch angle benefits to phases at lower instantaneous pitch angle by 'rolling' on the upper surface of the blade $\left(t=1 / 4 f_{p}\right)$. The LEV is much stronger than in the $\left(\alpha_{m}=10^{\circ}, \Delta \alpha=5^{\circ}, f_{p} / f_{r}=18\right)$ case though, and thus has a much stronger influence on circulatory forces.

To conclude, the ratio between LEV formation and pitching frequencies should be wisely tuned to best benefit from dynamic stall and LEV 'surface-rolling'. However, because LEV formation rate varies along the blade span and because nonlinear effects arise from highly unsteady separated flow (and its coupling with blade pitching motion), a specific pitching frequency may enhance aerodynamic performance in a given blade section while being detrimental to it in another blade section. This is different to observations made for group $A$ and is presumably responsible for the non-linear trend in $\overline{C_{T}}$ and $\overline{C_{P_{r}}}$ as a function of $f_{p} / f_{r}$ observed in Fig. 11.

\section{Conclusion}

We numerically investigated the influence of blade pitching motion on rotor aerodynamics. Toward that end, we performed a parametrical study by systematically varying pitching motion variables, i.e. blade mean pitch angle, pitching amplitude and pitching frequency, leading to a total of 74 test cases. Results show that an increase in rotational efficiency and thrust can be obtained depending on the variable setting.

First, it is shown that an increase in rotational efficiency and thrust can be obtained for cases with low to moderate mean pitch angles $\alpha_{m}$ (e.g. $5^{\circ}, 10^{\circ}$, and $15^{\circ}$ ), low pitching amplitudes $\Delta \alpha\left(<5^{\circ}\right)$ and high pitching-to-rotation frequency ratios $f_{p} / f_{r}(>8)$. These cases (referred to as group $\mathrm{A}$ in the paper) are found to promote thrust via the formation of a leading edge vortex (LEV) and subsequent LEV rolling on the upper surface of the blade, while limiting and potentially decreasing blade rotational torque via reversed von Karman street phenomenon. The occurrence of such phenomena depends on the reduced 
pitching frequency, hence local blade radius. Although this intrinsically induces a three-dimensional flow, formation processes are found to be quasi-two-dimensional processes. Indeed, reversed von Karman street manifests at local reduced frequencies above 5 , which is in line with previous two-dimensional studies. On the other hand, reduction in rotational torque is associated with an increase in pitching torque, such that the global aerodynamic efficiency is reduced. This corroborates previous conclusions drawn by two-dimensional studies in that reversed von Karman street is an inefficient process. Still, micro-air vehicles relying on pitching-rotor concepts could potentially benefit from it. A solution that is currently under investigation is to implement azimuthally distributed permanent magnetic fields that induce a periodic pitching of the blade as the latter rotates through the fields. Evaluating the relevancy of such a solution goes beyond the scope of rotor aerodynamics though, and needs to be addressed in the framework of an integrative-oriented study.

Second, it is shown that an increase in thrust can be obtained without global efficiency penalties for cases with high mean pitch angles $\alpha_{m}$ (e.g. $40^{\circ}$ ), moderate pitching amplitudes $\Delta \alpha$ (e.g. $15^{\circ}$ ) and moderate pitching-to-rotation frequency ratios $f_{p} / f_{r}$ (e.g. 5). These cases (referred to as group B in the paper) are found to promote thrust via dynamic stall and the formation of a strong LEV and subsequent LEV rolling on the upper surface of the blade. Due to the intrinsic unsteadiness of massive flow separation at high pitch angles, strong non-linear effects arise that make group B fundamentally different from group A. The resulting complexity of the flow makes quasi-two-dimensional approaches most likely inappropriate for these specific cases.

Overall, the analysis demonstrates that blade pitching can be beneficial to the aerodynamic performance of micro-air vehicles. Efficiency and thrust can be significantly modified, thereby increasing the global flight envelope and potentially promoting maneuverability. However, a clear outcome is that further improvements could be obtained by modifying the blade planform in order to ensure constant local reduced frequency along the blade span. This will be the scope of a future work.

\section{Appendix A. Convergence tests}

Preliminary tests are performed to ensure that the computational results are converged with respect to the number of cells and the time step used for the spatial and temporal discretizations respectively. Since the main goal of the present study is to cover a large parameter space in order to provide a global overview of pitching-rotor aerodynamics, our convergence criterion is defined such that reasonable accuracy is achieved while limiting the computational cost (i.e. the convergence criterion is not too restrictive).

As such, we here posit that a solution is converged if decreasing the typical cell size and time step do not result in a variation of the mean thrust and torque of more than $5 \%$. The mean thrust and torque values are computed by averaging the instantaneous thrust and torque over a rotation period once the flow has reached a periodic state. Again, we here posit that a solution has reached periodicity if computing the mean thrust and torque over any of the following rotation periods does not result in a variation of more than $5 \%$.

In order to perform these preliminary tests, four computational meshes and three computational time steps are considered. Table A1 provides the resulting computational setups, where the top row indicates the total number of cells in thousands and the first column indicates the time step.

Since the convergence tests cannot be performed for all cases, we consider four specific cases that are believed to be representative of the explored parameter space.

The first case has an $\alpha_{m}$ set to $15^{\circ}$ and corresponds to the non-pitching case that provides the highest efficiency. The second case has a similar $\alpha_{m}=15^{\circ}$ but pitches with pitching parameters $\Delta \alpha=15^{\circ}$ and $f_{p} / f_{r}=4$. The third case has an $\alpha_{m}$ set to $40^{\circ}$ and corresponds to the non-pitching case that provides the largest lift. The fourth case has a similar $\alpha_{m}=40^{\circ}$ but pitches with pitching parameters $\Delta \alpha=15^{\circ}$ and $f_{p} / f_{r}=4$.

The results obtained for the first, second, third and fourth cases are shown in Figs. A1-A4 respectively. These figures depict the percentage variations in mean thrust and torque obtained with respect to the reference setup at each rotation period and for all configurations listed in Table A1. The reference setup corresponds to the square symbols, which therefore lie on the zero axis. This representation helps analyze convergence with respect to spatial and temporal resolutions. In addition, for the reference setup, the percentage variations in mean thrust and torque obtained at each rotation period, with respect to the solution obtained at a given period (that ensures periodicity to be reached) are computed and displayed using the error bars. This representation helps analyze convergence with respect to transient phases (or flow periodicity). Dashdotted lines indicate the $5 \%$ bounds of the convergence criterion.

Table A1

Computational meshes and time steps used for convergence tests.

\begin{tabular}{lcccc}
\hline Time step & $600 \mathrm{k}$ & $1000 \mathrm{k}$ & $5000 \mathrm{k}$ & $9000 \mathrm{k}$ \\
\hline $360 / f_{r}$ & $\times$ & & + & \\
$720 / f_{r}$ & & 0 & $\square$ & $\diamond$ \\
$1440 / f_{r}$ & & & $\Delta$ & \\
\hline
\end{tabular}



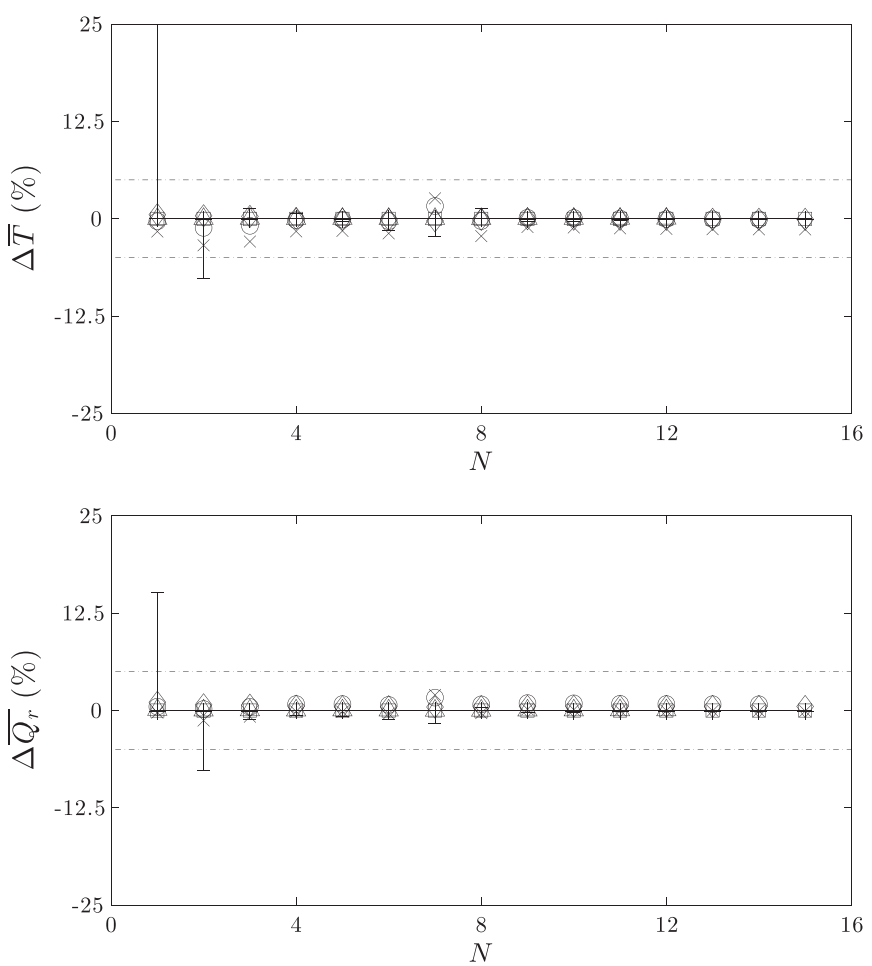

Fig. A1. Percentage variations in mean thrust (top) and torque (bottom) with respect to the reference setup as a function of the rotation period. Non-pitching $\alpha_{m}=15^{\circ}$ case. Symbol meanings are indicated in Table A1.
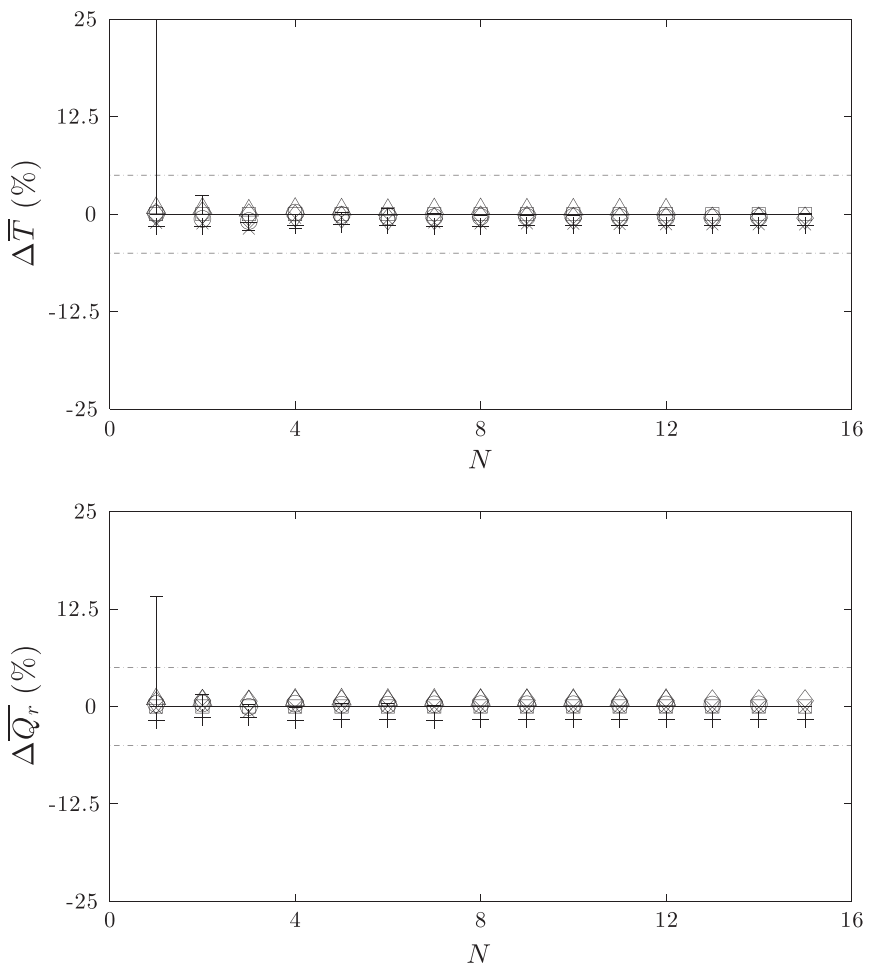

Fig. A2. Percentage variations in mean thrust (top) and torque (bottom) with respect to the reference setup as a function of the rotation period. Pitching $\alpha_{m}=15^{\circ}, \Delta \alpha=15^{\circ}$ and $f_{p} / f_{r}=4$ case. Symbol meanings are indicated in Table A1. 

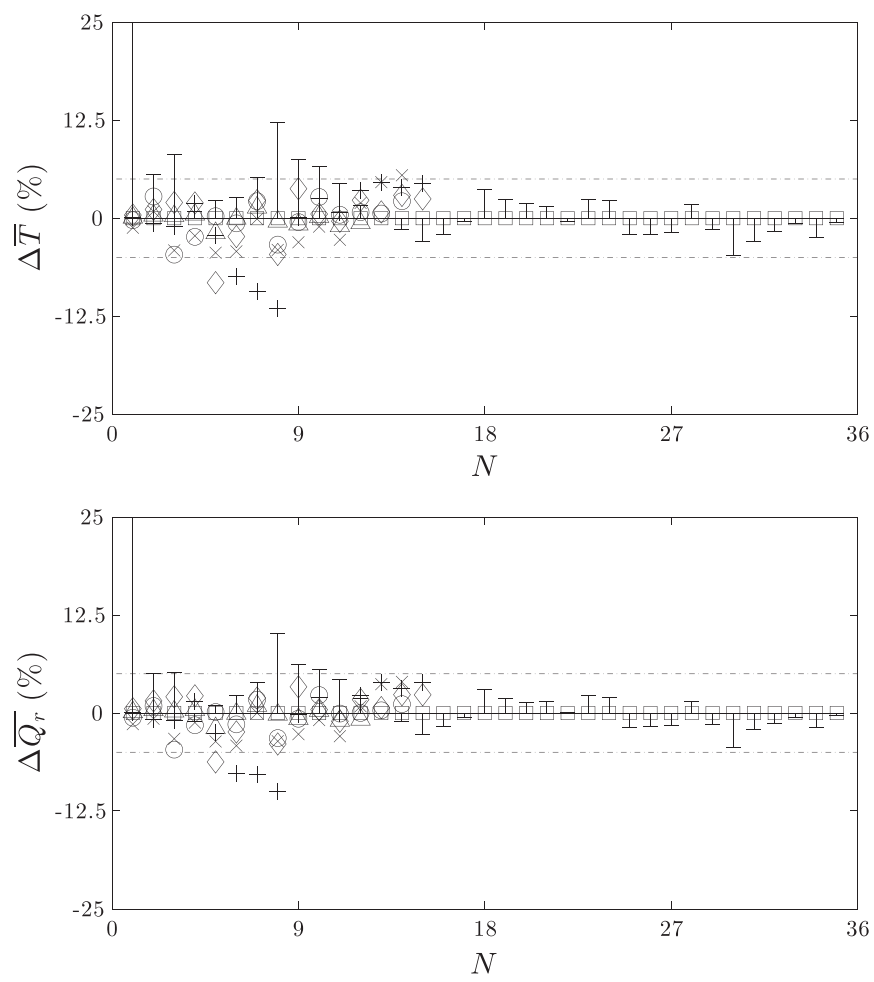

Fig. A3. Percentage variations in mean thrust (top) and torque (bottom) with respect to the reference setup as a function of the rotation period. Non-pitching $\alpha_{m}=40^{\circ}$ case. Symbol meanings are indicated in Table A1.
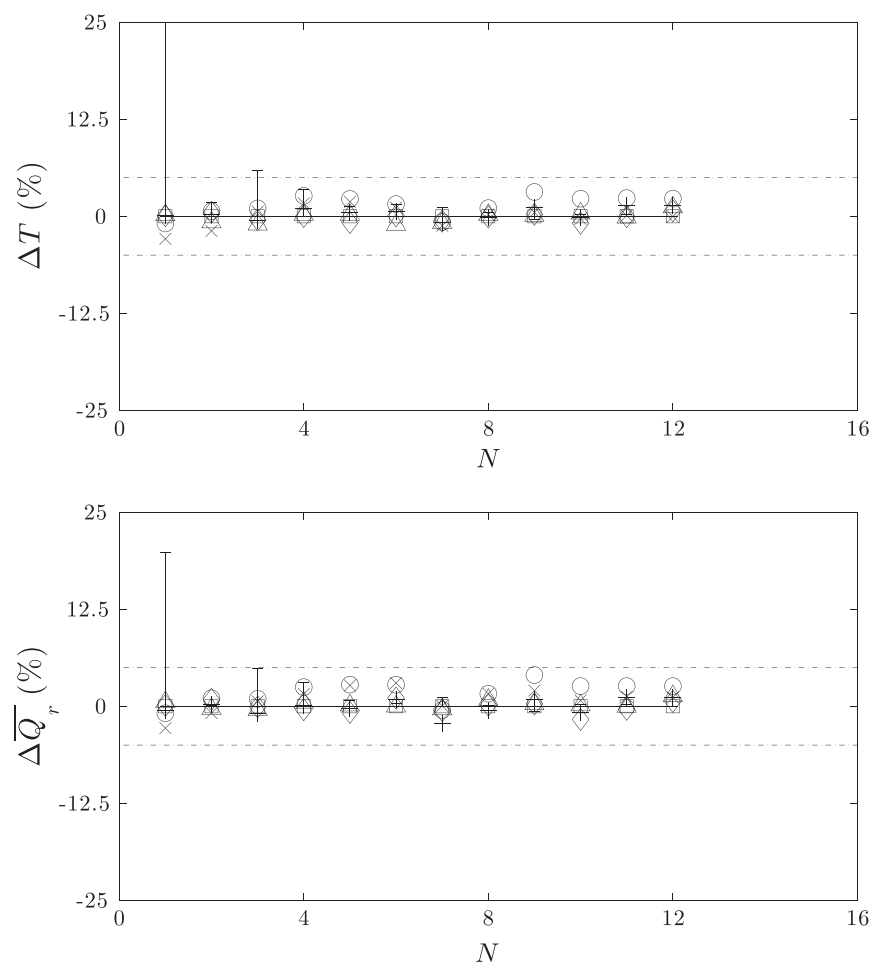

Fig. A4. Percentage variations in mean thrust (top) and torque (bottom) with respect to the reference setup as a function of the rotation period. Pitching $\alpha_{m}=40^{\circ}, \Delta \alpha=15^{\circ}$ and $f_{p} / f_{r}=4$ case. Symbol meanings are indicated in Table A1. 
It can be seen that the first two cases are not very sensitive to changes in spatial and temporal resolutions, within the range tested. Symbols are hardly distinguishable and convergence is even achieved for the lowest spatial and temporal resolutions within $2 \%$ bounds. In addition, it can be seen that periodicity is reached very quickly, for $N \geq 3$ within $5 \%$ bounds, and for $N \geq 8$ within $2 \%$ bounds. On the contrary, the third case is much more sensitive to changes in spatial and temporal resolutions. Such a sensitivity is due to the occurrence of natural vortex shedding, at least on the outboard region of the blade, which may not be locked on the rotation frequency. Indeed, slight variations in the prediction of natural shedding frequency may have a greater impact on aerodynamic loads as the blades encounter their own wake. Accordingly, although the loads of the reference setup do reach periodicity within $5 \%$ bounds for $N \geq 11$, they do not reach periodicity within $2 \%$ bounds. Finally, it can be seen from the fourth case that sensitivity to computational parameters and variations from one rotation period to another are significantly damped as a pitching motion is imposed. Here, convergence with respect to spatial and temporal resolutions is achieved for the reference setup within $2 \%$ bounds and periodicity is reached for $N \geq 5$ within $2 \%$ bounds.

From these cases, it is believed that most of the cases addressed in this study are converged with respect to spatial and temporal resolutions within $2 \%$ bounds and that only few cases at high angles of attack and low frequency ratios are converged within $5 \%$ bounds. Furthermore, while convergence with respect to spatial and temporal resolutions cannot be checked for all cases, periodicity is automatically checked for all cases. It should be emphasized here that periodicity is defined with respect to the rotation period, which is only valid if the pitching-to-rotation frequency is an integer. All cases in this study satisfies this condition.

\section{References}

Bohorquez, F., Pines, D.J., 2002. Design and development of a biomimetic device for micro air vehicles. In: SPIE's 9th Annual International Symposium on Smart Structures and Materials, pp. 503-517.

CD-Adapco, 2013. User Guide Star-CCM+ Version 8.02.

Dickinson, M.H., Lehmann, F.O., Sane, S.P., 1999. Wing rotation and the aerodynamic basis of insect flight. Science 284, 1954-1960.

Eloy, C., 2012. Optimal Strouhal number for swimming animals. Journal of Fluids and Structures 30, $205-218$.

Eldredge, J.D., Wang, C., Ol, M.V., 2009. A Computational Study of a Canonical Pitch-up, Pitch-Down Wing Maneuver. AIAA Paper 2009-3687.

Ferziger, J.H., Peric, M., 2002. Computational Methods for Fluid Dynamics, 3rd rev. ed. Springer-Verlag, Berlin.

Fitchett, B., Chopra, I., 2007. A biologically inspired flapping rotor for micro air vehicles. In: AHS International Specialists Meeting on Unmanned Rotorcraft, January 23-25, 2007.

Theodoor, van Holten., 2002. A single rotor without reaction torque: a violation of Newton's laws or feasible? In: 28th European Rotorcraft Forum.

Jacobs, E.N., Sherman, A., 1937. Airfoil section characteristics as affected by variations of the Reynolds number. NACA Report 586.

Jardin, T., David, L., 2014. Spanwise gradients in flow speed help stabilize leading-edge vortices on revolving wings. Physical Review E $90,013011$.

Jardin, T., David, L., 2015. Coriolis effects enhance lift on revolving wings. Physical Review E 91, 031001.

Jardin, T., Prothin, S., Garcia Magana, C., 2015. Aerodynamics of micro-rotor in confined environment. In: AHS International 71st Annual Forum \& Technology Display.

Jeong, J., Hussain, F., 1995. On the identification of a vortex. Journal of Fluid Mechanics 285, 69-94.

Jones, K.D., Bradshaw, C.J., Papadopoulos, J., Platzer, M.F., 2005. Bio-inspired design of flapping-wing micro air vehicles. Aeronautical Journal 109, 385-394.

Keennon, M., Klingebiel, K., Won, H., Andriukov, A., 2012. Development of the nano hummingbird: a tailless flapping wing micro air vehicle. In: 50th AIAA Aerospace Sciences Meeting Including the New Horizons Forum and Aerospace Exposition, January 9-12, 2012.

Kruyt, J.W., van Heijst, G.F., Altshuler, D.L., Lentink, D., 2015. Power reduction and the radial limit of stall delay in revolving wings of different aspect ratio. Journal of The Royal Society Interface 12, 20150051.

Lentink, T., Dickinson, M.H., 2009. Rotational accelerations stabilize leading edge vortices on revolving fly wings. Journal of Experimental Biology 212 2705-2719.

Mackowski, A.W., Williamson, C.H.K., 2015. Direct measurement of thrust and efficiency of an airfoil undergoing pure pitching. Journal of Fluid Mechanics 765, 524-543.

McCroskey, W.J., 1982. Unsteady airfoils. Annual Review of Fluid Mechanics 14, 285-311.

Rhie, C.M., Chow, W.L., 1983. Numerical study of the turbulent flow past an airfoil with trailing edge separation. AIAA Journal $21,1525-1532$.

Sarkar, S., Venkatraman, K., 2006. Numerical simulation of thrust generating flow past a pitching airfoil. Computers \& Fluids 35, 16-42.

Spalart, P.R., Allmaras, S.R., 1992. A one-equation turbulence model for aerodynamic flows. In: AIAA 30th Aerospace Sciences Meeting and Exhibit, 0439.

Visbal, M.R., 1990. Dynamic stall of a constant-rate pitching airfoil. Journal of Aircraft 27, 400-407.

Wang, Z., 2000. Vortex shedding and frequency selection in flapping flight. Journal of Fluid Mechanics 410, $323-341$.

Wood, R.J., 2008. The first takeoff of a biologically inspired at-scale robotic insect. IEEE Transactions on Robotics 24, 341-347. 